\title{
LOWER ESTIMATES FOR $p$-MODULI AND SOBOLEV CLASS MAPPINGS
}

\author{
R. R. SALIMOV
}

\begin{abstract}
Finite distortion homeomorphisms on the plane are studied with the help of the moduli techniques. On that basis, a problem that dates back to M. A. Lavrent'ev is solved; this problem concerns estimating the area of the image of a disk under the mappings mentioned above. The asymptotic behavior of such mappings at a point is studied. A condition ensuring the finite Lipschitz property is found.
\end{abstract}

\section{$\S 1$. INTRODUCTION}

In this paper, we study mappings that obey certain lower moduli estimates the theory of which applies to the Sobolev $W_{\text {loc }}^{1,1}$ class mappings with finite distortion. We restrict ourselves to the plane case.

We recall some definitions. Let $D$ be a domain on the complex plane, i.e., a connected open subset of $\mathbb{C}$. A continuous mapping $\gamma$ of an open set $\Delta$ on the real axis $\mathbb{R}$ or on a circle into $D$ is called a dashed line (see, e.g., [2, 6.3]). The name "dashed line" is motivated by the fact that any open set $\Delta$ in $\mathbb{R}$ is composed of at most countably many mutually disjoint intervals.

Let a family $\Gamma$ of dashed lines $\gamma$ be given in $\mathbb{C}$. A Borel function $\rho: \mathbb{C} \rightarrow[0, \infty]$ is said to be admissible for $\Gamma$ (we write $\rho \in \operatorname{adm} \Gamma$ ) if

$$
\int_{\gamma} \rho(z)|d z| \geq 1
$$

for all $\gamma \in \Gamma$.

Let $p \in(1, \infty)$. By the $p$-module of the family $\Gamma$ we mean the quantity

$$
\mathcal{M}_{p}(\Gamma)=\inf _{\rho \in \operatorname{adm} \Gamma} \int_{\mathbb{C}} \rho^{p}(z) d m(z)
$$

We say that a property $P$ is fulfilled for $p$-a.e. (almost every) $\gamma \in \Gamma$ if the subfamily of all lines in $\Gamma$ for which $P$ fails has $p$-module equal to zero (see [3]). Also, we say that a Lebesgue measurable function $\rho: \mathbb{C} \rightarrow[0, \infty]$ is extendedly admissible and write $\rho \in \operatorname{ext}_{p} \operatorname{adm} \Gamma$ if (11) is true for $p$-a.e. $\gamma \in \Gamma$ (see, e.g., [2, Subsection 9.2]).

The next notion is motivated by Gehring's ring definition of quasiconformity. Given two domains $D$ and $D^{\prime}$ in $\mathbb{C}$, a point $z_{0} \in D$, and a measurable function $Q: D \rightarrow(0, \infty)$, we say that a homeomorphism $f: D \rightarrow D^{\prime}$ is a lower $Q$-homeomorphism relative to the $p$-module at $z_{0}$ if

$$
\mathcal{M}_{p}(f \Sigma) \geq \inf _{\rho \in \operatorname{ext}_{p} \operatorname{adm} \Sigma\left(z_{0}, r_{1}, r_{2}\right)} \int_{R} \frac{\rho^{p}(z)}{Q(z)} d m(z)
$$

2010 Mathematics Subject Classification. Primary 30C62; Secondary 46E35.

Key words and phrases. $p$-Molude, p-capacity, mappings with finite distortion, finite Lipschitzian property. 
for any ring

$$
R=R\left(z_{0}, r_{1}, r_{2}\right)=\left\{z \in \mathbb{C}: r_{1}<\left|z-z_{0}\right|<r_{2}\right\}, \quad 0<r_{1}<r_{2}<d_{0},
$$

where $d_{0}=\operatorname{dist}\left(z_{0}, \partial D\right)$, and $\Sigma=\Sigma\left(z_{0}, r_{1}, r_{2}\right)$ denotes the family of all circles $C\left(z_{0}, r\right)=$ $\left\{z \in \mathbb{C}: z-z_{0} \mid=r\right\}, r \in\left(r_{1}, r_{2}\right)$.

Historically, prior to the lower $Q$-homomorphisms relative to the $p$-module, the notion of a $Q$-homomorphism had arisen, which was studied in [5, 6, 7, 8]. Also, the $Q$-homomorphisms with branching points were explored in [9, 10, 11, 12, 13.

The theory of lower $Q$-homomorphisms relative to the $p$-module, devoloped in this paper, applies, in particular, to the mappings quasiconformal in the mean (see [14, 15]), and to the so-called $(p, q)$-quasiconformal mappings, which were employed in the study of Yu. Rechetnyak's problem on composition of Sobolev class functions, see [17, 18, 19.

In the papers [20, 21, 22], the theory of lower $Q$-homeomorphisms was applied to the study of local and boundary behavior of homeomorphic solutions with generalized derivatives and to the Dirichlet problem for the Beltrami equations with degeneration. Also, this theory can be applied to finite distortion mappings belonging to the OrliczSobolev class $W_{\text {loc }}^{1, \varphi}$ under the Calderón condition, and, in particular, to the Sobolev class $W_{\text {loc }}^{1, p}$ with $p>n-1$, see [23, 24, 25].

\section{$\S 2$. ON THE CAPACITY OF A CONDENSER}

As in [26], by a condenser we mean a pair $\mathcal{E}=(A, C)$, where $A \subset \mathbb{C}$ is an open set and $C$ is a nonempty compact set lying in $A$. Such $\mathcal{E}$ is called a ring condenser if $\Re=A \backslash C$ is a ring, i.e., a domain whose complement $\overline{\mathbb{C}} \backslash \mathfrak{R}$ has precisely two components. A condenser is said to be bounded it so is the set $A$. Also, we say that $\mathcal{E}=(A, C)$ lies in a domain $D$ if $A \subset D$. Obviously, if $f: D \rightarrow \mathbb{C}$ is a continuous and open mapping and $\mathcal{E}=(A, C)$ is a condenser in $D$, then $(f A, f C)$ is a condenser in $D$; we write $f \mathcal{E}=(f A, f C)$.

Let $\mathcal{E}=(A, C)$ be a condenser. We denote by $\mathcal{C}_{0}(A)$ the set of continuous functions $u: A \rightarrow \mathbb{R}^{1}$ with compact support, and by $\mathcal{W}_{0}(\mathcal{E})=\mathcal{W}_{0}(A, C)$ the family of all nonnegative functions $u: A \rightarrow \mathbb{R}^{1}$ such that

1) $\left.u \in \mathcal{C}_{0}(A), 2\right) u(x) \geq 1$ for $x \in C$, and 3) $u$ belongs to the class ACL; we put

$$
|\nabla u|=\sqrt{\left(\frac{\partial u}{\partial x}\right)^{2}+\left(\frac{\partial u}{\partial y}\right)^{2}} .
$$

For $p \geq 1$, the quantity

$$
\operatorname{cap}_{p} \mathcal{E}=\operatorname{cap}_{p}(A, C)=\inf _{u \in \mathcal{W} \mathcal{W}_{0}(\mathcal{E})} \int_{A}|\nabla u|^{p} d m(z)
$$

is called the $p$-capacity of the condenser $\mathcal{E}$. In what follows, we shall use the identity

$$
\operatorname{cap}_{p} \mathcal{E}=\mathcal{M}_{p}(\Delta(\partial A, \partial C ; A \backslash C)),
$$

where, for sets $\mathcal{F}_{1}, \mathcal{F}_{2}$, and $\mathcal{F}$ in $\mathbb{C}, \Delta\left(\mathcal{F}_{1}, \mathcal{F}_{2} ; \mathcal{F}\right)$ denotes the family of all continuous curves that connect $\mathcal{F}_{1}$ and $\mathcal{F}_{2}$ in $\mathcal{F}$ (see [27] and [28, 29]). In the context of the theory of mappings, capacities are well presented in the book [30].

For $1<p \leq 2$, we have the estimate

$$
\operatorname{cap}_{p} \mathcal{E} \geq \gamma_{p} \frac{d(C)^{p}}{m(A)^{p-1}}
$$

where $d(C)$ is the diameter of the compact set $C, m(A)$ is the Lebesgue measure of $A$, and $\gamma_{p}$ is a positive constant depending only on $p$ (see [15, Proposition 6]). 
Moreover, the following estimate is known for $1<p<2$ :

$$
\operatorname{cap}_{p} \mathcal{E} \geq 2 \pi^{\frac{p}{2}}\left(\frac{2-p}{p-1}\right)^{p-1}[m(C)]^{\frac{2-p}{2}}
$$

(see, e.g., [31, 1.4]).

Also, for $p \geq 1$ we have

$$
\operatorname{cap}_{p} \mathcal{E} \geq \frac{[\inf l(\sigma)]^{p}}{[m(A \backslash C)]^{p-1}},
$$

where $l(\sigma)$ stands the length of a curve; here $\sigma$ is a smooth (infinitely differentiable) curve that is the boundary $\sigma=\partial U$ of a bounded open set $U$ containing $C$ and included in $A$ together with its closure $\bar{U}$; the infimum in (10) is taken over all such curves $\sigma$ (see [15. Proposition 5]).

\section{$\S 3$. A CRITERION FOR LOWER $Q$-HOMEOMORPhisms RElative TO THE $p$-MOdule}

In the sequel, we adopt the following standard agreements: $a / \infty=0$ for $a \neq \infty$, $a / 0=\infty$ for $a>0$, and $0 \cdot \infty=0$ (see, e.g., [32).

Let $(X, \mu)$ be a measurable space with finite measure $\mu$, and let $\varphi: X \rightarrow \mathbb{R}$ be a measurable function. For any measurable set $E \subset X$, we denote

$$
f_{E} \varphi(x) d \mu(x)=\frac{1}{\mu(E)} \int_{E} \varphi(x) d \mu(x) .
$$

Before proving the main lemma on lower $Q$-homeomorphisms relative to the $p$-module, we present the auxiliary Lemma 2.1 from [33] (see [2, Lemma 9.2]).

Lemma 1. Let $(X, \mu)$ be a measurable space with finite measure $\mu$, let $q \in(1, \infty)$, and let $\varphi: X \rightarrow(0, \infty)$ be a measurable function. Put

$$
I(\varphi, q)=\inf _{\alpha} \int_{X} \varphi \alpha^{q} d \mu
$$

where the infimum is taken over all measurable functions $\alpha: X \rightarrow[0, \infty]$ such that

$$
\int_{X} \alpha d \mu=1
$$

Then

$$
I(\varphi, q)=\left[\int_{X} \varphi^{-\lambda} d \mu\right]^{-\frac{1}{\lambda}}
$$

where

$$
\lambda=\frac{q^{\prime}}{q}, \quad \frac{1}{q}+\frac{1}{q^{\prime}}=1,
$$

i.e., $\lambda=1 /(q-1) \in(0, \infty)$. Moreover, the infimum in (12) is only attained at the function

$$
\alpha_{0}=\gamma \cdot \varphi^{-\lambda}
$$

where

$$
\gamma=\left(\int_{X} \varphi^{-\lambda} d \mu\right)^{-1}
$$

The statement below is a criterion for a homeomorphism in $\mathbb{C}$ to be a lower $Q$-homeomorphism relative to the $p$-module at a point. 
Lemma 2. Let $D$ be a domain in $\mathbb{C}$, let $z_{0} \in D$, and let $Q: D \rightarrow(0, \infty)$ be a measurable function. A homeomorphism $f: D \rightarrow \mathbb{C}$ is a lower $Q$-homeomorphism at the point $z_{0}$ relative to the $p$-module for $p>1$ if and only if

$$
\mathcal{M}_{p}(f \Sigma) \geq \int_{r_{1}}^{r_{2}} \frac{d r}{\|Q\|_{\frac{1}{p-1}}\left(z_{0}, r\right)}
$$

for all $0<r_{1}<r_{2}<d_{0}=\operatorname{dist}\left(z_{0}, \partial D\right)$, where

$$
\|Q\|_{\frac{1}{p-1}}\left(z_{0}, r\right)=\left(\int_{C\left(z_{0}, r\right)} Q^{\frac{1}{p-1}}(z)|d z|\right)^{p-1}
$$

$\Sigma=\Sigma\left(z_{0}, r_{1}, r_{2}\right)$ is the family of all circles $C\left(z_{0}, r\right)=\left\{z \in \mathbb{C}:\left|z-z_{0}\right|=r\right\}, r \in\left(r_{1}, r_{2}\right)$. The infimum in (3) is only attained at the function

$$
\rho_{0}(z)=\frac{Q(z)}{\|Q\|_{\frac{1}{p-1}}\left(z_{0},\left|z-z_{0}\right|\right)} .
$$

Proof. For any function $\rho(z) \in \operatorname{ext}_{p} \operatorname{adm} \Sigma\left(z_{0}, r_{1}, r_{2}\right)$ we have

$$
A_{\rho}(r):=\int_{C\left(z_{0}, r\right)} \rho(z)|d z| \neq 0 \text { a.e., }
$$

and this quantity is measurable with respect to the parameter $r$ by, e.g., the Fubini theorem. Thus, we may replace the admissibility condition (1) with the requirement $A_{\rho}(r) \equiv 1$, a.e., and

$$
\inf _{\rho \in \operatorname{ext}_{p} \operatorname{adm} \Sigma} \int_{R\left(z_{0}, r_{1}, r_{2}\right)} \frac{\rho^{p}(z)}{Q(z)} d m(z)=\int_{r_{1}}^{r_{2}}\left(\inf _{\alpha \in \mathfrak{I}(r)} \int_{C\left(z_{0}, r\right)} \frac{\alpha^{p}(z)}{Q(z)}|d z|\right) d r,
$$

where $\mathfrak{I}(r)$ is the set of all measurable functions $\alpha(z)$ on the circle $C\left(z_{0}, r\right)$ such that

$$
\int_{C\left(z_{0}, r\right)} \alpha(z)|d z|=1
$$

Now, Lemma 2 follows from Lemma 1 for $X=C\left(z_{0}, r\right), \mu$ is the arclength on $C\left(z_{0}, r\right)$, and $\varphi=\left.\frac{1}{Q}\right|_{C\left(z_{0}, r\right)}$.

Thus, inequality (18) is sharp for the lower $Q$-homeomorphisms relative to the $p$-module. The lemma is proved.

Inequality (18) can be written in another form, which will sometimes be more convenient.

Lemma 3. Let $Q: D \rightarrow(0, \infty)$ be a measurable function, and let $f: D \rightarrow D^{\prime}$ be a lower $Q$-homeomorphism at a point $z_{0} \in D$ relative to the $p$-module with $p>1$. Then for any $0<r_{1}<r_{2}<d_{0}=\operatorname{dist}\left(z_{0}, \partial D\right)$ we have

$$
\mathcal{M}_{p^{\prime}}\left(f\left(\Delta\left(C_{1}, C_{2}, D\right)\right)\right) \leq\left(\int_{r_{1}}^{r_{2}} \frac{d r}{\|Q\|_{\frac{1}{p-1}}\left(z_{0}, r\right)}\right)^{-\frac{1}{p-1}}
$$

where $\frac{1}{p}+\frac{1}{p^{\prime}}=1$ and

$$
\|Q\|_{\frac{1}{p-1}}\left(z_{0}, r\right)=\left(\int_{C\left(z_{0}, r\right)} Q^{\frac{1}{p-1}}(z)|d z|\right)^{p-1} .
$$

Proof. Indeed, let $0<r_{1}<r_{2}<d\left(z_{0}, \partial D\right)$, and let $C_{i}=C\left(z_{0}, r_{i}\right), i=1,2$. By the Hesse-Ziemer inequality (see e.g., 27] and [34]), we have

$$
\mathcal{M}_{p^{\prime}}\left(f\left(\Delta\left(C_{1}, C_{2}, D\right)\right)\right) \leq \frac{1}{\mathcal{M}_{p}^{\frac{1}{p-1}}\left(f\left(\Sigma\left(z_{0}, r_{1}, r_{2}\right)\right)\right)},
$$


because $f\left(\Sigma\left(z_{0}, r_{1}, r_{2}\right)\right) \subset \Sigma\left(f\left(C_{1}\right), f\left(C_{2}\right), f(D)\right)$, where $\Sigma\left(z_{0}, r_{1}, r_{2}\right)$ denotes the set of all circles with center $z_{0}$ lying between $C_{1}$ and $C_{2}$, and $\Sigma\left(f\left(C_{1}\right), f\left(C_{2}\right), f(D)\right)$ is formed by all curves in $f(D)$ that separate $f\left(C_{1}\right)$ and $f\left(C_{2}\right)$. By (26), from Lemma 3 if follows that

$$
\mathcal{M}_{p^{\prime}}\left(f\left(\Delta\left(C_{1}, C_{2}, D\right)\right)\right) \leq\left(\int_{r_{1}}^{r_{2}} \frac{d r}{\|Q\|_{\frac{1}{p-1}}\left(z_{0}, r\right)}\right)^{-\frac{1}{p-1}} .
$$

Lemma 4. Let $Q: D \rightarrow(0, \infty)$ be a measurable function such that $\|Q\|_{\frac{1}{p-1}}\left(z_{0}, r\right) \neq 0$ for a.e. $r \in\left(0, d_{0}\right), d_{0}=\operatorname{dist}\left(z_{0}, \partial D\right)$, and let $f: D \rightarrow D^{\prime}$ be a lower $Q$-homeomorphism at $z_{0} \in D$ relative to the $p$-module with $p>1$. Put

$$
\eta_{0}(t)=1 / I \cdot\|Q\|_{\frac{1}{p-1}}\left(z_{0}, t\right)
$$

where

$$
I=I\left(z_{0}, r_{1}, r_{2}\right)=\int_{r_{1}}^{r_{2}} \frac{d r}{\|Q\|_{\frac{1}{p-1}}\left(z_{0}, r\right)}
$$

Then

$$
\begin{aligned}
I^{-\frac{1}{p-1}} & =\int_{R\left(z_{0}, r_{1}, r_{2}\right)} Q^{\frac{1}{p-1}}(z) \cdot \eta_{0}^{p^{\prime}}\left(\left|z-z_{0}\right|\right) d m(z) \\
& \leq \int_{R\left(z_{0}, r_{1}, r_{2}\right)} Q^{\frac{1}{p-1}}(z) \cdot \eta^{p^{\prime}}\left(\left|z-z_{0}\right|\right) d m(z)
\end{aligned}
$$

for any measurable function $\eta:\left(r_{1}, r_{2}\right) \rightarrow[0, \infty]$ such that

$$
\int_{r_{1}}^{r_{2}} \eta(r) d r=1
$$

Proof. If $I=\infty$, then the left-hand side of (30) is zero, making the inequality obvious. If $I=0$, then $\|Q\|_{\frac{1}{p-1}}\left(z_{0}, r\right)=\infty$ for a.e. $r \in\left(r_{1}, r_{2}\right)$, so that the two sides of (30) are infinite by the Fubini theorem. Now, let $0<I<\infty$. Then $\|Q\|_{\frac{1}{p-1}}\left(z_{0}, r\right) \neq 0$ and $\eta_{0}(r) \neq \infty$ a.e. in $\left(r_{1}, r_{2}\right)$. Putting

$$
\alpha(r)=\eta(r) \cdot\|Q\|_{\frac{1}{p-1}}\left(z_{0}, r\right)
$$

and

$$
\omega(r)=\left[\|Q\|_{\frac{1}{p-1}}\left(z_{0}, r\right)\right]^{-1},
$$

and using the standard agreements, we see that $\eta(r)=\alpha(r) \omega(r)$ a.e. in $\left(r_{1}, r_{2}\right)$, and that

$$
C:=\int_{R} Q^{\frac{1}{p-1}}(z) \cdot \eta^{p^{\prime}}\left(\left|z-z_{0}\right|\right) d m(z)=\int_{r_{1}}^{r_{2}} \alpha^{p^{\prime}}(r) \omega(r) d r .
$$

Applying the Jensen inequality with weight (see [35, Theorem 2.6.2]) to the convex function $\varphi(t)=t^{p^{\prime}}$ defined on the interval $\Omega=\left(r_{1}, r_{2}\right)$ with the probability measure

$$
\nu(E)=\frac{1}{I} \int_{E} \omega(r) d r
$$

we obtain the inequality

$$
\left(f \alpha^{p^{\prime}}(r) \omega(r) d r\right)^{\frac{1}{p^{\prime}}} \geq f \alpha(r) \omega(r) d r=\frac{1}{I}
$$

we have also used the fact that $\eta(r)=\alpha(r) \omega(r)$ satisfies (31). Thus,

$$
C \geq I^{-\frac{1}{p-1}}
$$

which proves (30). 
Combining Lemmas 3 and 4, we get the following statement.

Lemma 5. Let $Q: D \rightarrow(0, \infty)$ be a measurable function such that $\|Q\|_{\frac{1}{p-1}}\left(z_{0}, r\right) \neq 0$ for a.e. $r \in\left(0, d_{0}\right), d_{0}=\operatorname{dist}\left(z_{0}, \partial D\right)$, and let $f: D \rightarrow D^{\prime}$ be a lower $Q$-homeomorphism at $z_{0} \in D$ relative to the $p$-module with $p>1$. Then for any $0<r_{1}<r_{2}<d_{0}$ we have

$$
\mathcal{M}_{p^{\prime}}\left(f\left(\Delta\left(C_{1}, C_{2}, D\right)\right)\right) \leq \int_{R\left(z_{0}, r_{1}, r_{2}\right)} Q^{\frac{1}{p-1}}(z) \cdot \eta^{p^{\prime}}\left(\left|z-z_{0}\right|\right) d m(z)
$$

for an arbitrary measurable function $\eta:\left(r_{1}, r_{2}\right) \rightarrow[0, \infty]$ satisfying (31).

Lemma 6. Let $Q: D \rightarrow(0, \infty)$ be a measurable function such that $\|Q\|_{\frac{1}{p-1}}\left(z_{0}, r\right) \neq 0$ for a.e. $r \in\left(0, d_{0}\right), d_{0}=\operatorname{dist}\left(z_{0}, \partial D\right)$, and let $f: D \rightarrow D^{\prime}$ be a lower $Q$-homeomorphism at $z_{0} \in D$ relative to the $p$-module with $p>1$. Then for any condenser $\mathcal{E}=\left(B\left(z_{0}, r_{2}\right)\right.$, $\left.\overline{B\left(z_{0}, r_{1}\right)}\right)$, where $0<r_{1}<r_{2}<d_{0}$, we have

$$
\operatorname{cap}_{p^{\prime}} f \mathcal{E} \leq \int_{R\left(z_{0}, r_{1}, r_{2}\right)} Q^{\frac{1}{p-1}}(z) \cdot \eta^{p^{\prime}}\left(\left|z-z_{0}\right|\right) d m(z)
$$

for an arbitrary measurable function $\eta:\left(r_{1}, r_{2}\right) \rightarrow[0, \infty]$ satisfying (31).

Proof. Consider the ring condenser

$$
\mathcal{E}=\left(B\left(z_{0}, r_{2}\right), \overline{B\left(z_{0}, r_{1}\right)}\right)
$$

with $0<r_{1}<r_{2}<d_{0}$ and the ring

$$
R=R\left(z_{0}, r_{1}, r_{2}\right)=\left\{z \in \mathbb{C}: r_{1}<\left|z-z_{0}\right|<r_{2}\right\} .
$$

Then $f \mathcal{E}=\left(f B\left(z_{0}, r_{2}\right), \overline{f B\left(z_{0}, r_{1}\right)}\right)$ is a ring condenser in $D^{\prime}$ and, by (7), we have

$$
\bigcap_{p^{\prime}}\left(f B\left(z_{0}, r_{2}\right), \overline{f B\left(z_{0}, r_{1}\right)}\right)=\mathcal{M}_{p^{\prime}}\left(\triangle\left(\partial f B\left(z_{0}, r_{2}\right), \partial f B\left(z_{0}, r_{1}\right) ; f R\right) .\right.
$$

Since $f$ is a homeomorphism, we also have the identity

$$
\triangle\left(\partial f B\left(z_{0}, r_{2}\right), \partial f B\left(z_{0}, r_{1}\right) ; f R\right)=f\left(\triangle\left(\partial B\left(z_{0}, r_{2}\right), \partial B\left(z_{0}, r_{1}\right) ; R\right)\right) .
$$

Now, estimate (39) follows from Lemma 5

\section{§4. Differentiability almost Everywhere}

Theorem 1. Let $D$ and $D^{\prime}$ be domains in $\mathbb{C}$, and let $f: D \rightarrow D^{\prime}$ be a $Q$-homeomorphism relative to the $p$-module with $p \geq 2$ and $Q(z) \in \mathrm{L}_{\mathrm{loc}}^{\frac{1}{p-1}}(D)$. Then $f$ is differentiable almost everywhere in $D$.

Consider the following set function defined on the Borel algebra $\mathfrak{B}$ in $D: \Phi(\mathfrak{B})=$ $m(f(\mathfrak{B}))$. The differentiability theorem for nonnegative subadditive set functions (see, e.g., [36, III.2.4]) shows that, for a.e. $z \in D$, the limit

$$
\varphi(z)=\lim _{\varepsilon \rightarrow 0} \frac{\Phi(B(z, \varepsilon))}{m(B(z, \varepsilon))},
$$

exists and is finite, where $B(z, \varepsilon)$ is the disk in $\mathbb{C}$ centered at $z \in \mathbb{C}$ and of radius $\varepsilon>0$. The quantity $\varphi(z)$ is called the volume derivative of the mapping $f$ at the point $z$.

For $z, \zeta \in D$, we put

$$
L(z, f)=\limsup _{\zeta \rightarrow z} \frac{|f(\zeta)-f(z)|}{|\zeta-z|} .
$$

Using the Rademacher-Stepanov theorem (see, e.g., [32]), we see that Theorem 1 will be proved it we cheek the following statement. 
Lemma 7. Let $D$ and $D^{\prime}$ be domains in $\mathbb{C}$, and let $f: D \rightarrow D^{\prime}$ be a lower $Q$-homeomorphism relative to the $p$-module with $Q \in \mathrm{L}_{\mathrm{loc}}^{\frac{1}{p-1}}(D)$. Then, for $p \geq 2$,

$$
L(z, f) \leq \gamma_{p} \varphi^{\frac{1}{p}}(z) Q^{\frac{1}{p}}(z) \text { for } z \in D,
$$

with a constant $\gamma_{p}$ depending only on $p$.

Proof. Consider the ring condenser

$$
\mathcal{E}=\left(B\left(z, r_{2}\right), \overline{B\left(z, r_{1}\right)}\right)
$$

with $0<r_{1}<r_{2}<d_{0}$ and the ring $R=R\left(z, r_{1}, r_{2}\right)=\left\{\zeta \in \mathbb{C}: r_{1}<|\zeta-z|<r_{2}\right\}$.

Observe that the function

$$
\eta(t)= \begin{cases}\frac{1}{r_{2}-r_{1}}, & t \in\left(r_{1}, r_{2}\right), \\ 0, & t \in \mathbb{R} \backslash\left(r_{1}, r_{2}\right),\end{cases}
$$

satisfies condition (31). Then, by Lemma 6, we have

$$
\operatorname{cap}_{p^{\prime}} f \mathcal{E} \leq\left(r_{2}-r_{1}\right)^{-p^{\prime}} \int_{R\left(z, r_{1}, r_{2}\right)} Q^{\frac{1}{p-1}}(\zeta) d m(\zeta) .
$$

Next, choosing $r_{1}=\varepsilon$ and $r_{2}=2 \varepsilon$ in (48), we get

$$
\operatorname{cap}_{p^{\prime}}(f B(z, 2 \varepsilon), \overline{f B(z, \varepsilon)}) \leq \varepsilon^{-p^{\prime}} \int_{B(z, 2 \varepsilon)} Q^{\frac{1}{p-1}}(\zeta) d m(\zeta)
$$

On the other hand, relation (8) implies

$$
\operatorname{cap}_{p^{\prime}}(f B(z, 2 \varepsilon), \overline{f B(z, \varepsilon)}) \geq\left(C_{p} \frac{d^{p}(\overline{f B(z, \varepsilon)})}{m(f B(z, 2 \varepsilon))}\right)^{\frac{1}{p-1}},
$$

where $C_{p}$ is a constant depending only on $p, d(A)$ is the diameter and $m(A)$ the Lebesgue measure of a set $A$ in $\mathbb{C}$.

Combining (49) and (50) yields

$$
\frac{d(\overline{f B(z, \varepsilon)})}{\varepsilon} \leq \gamma_{p}\left(\frac{m(f B(z, 2 \varepsilon))}{m(B(z, 2 \varepsilon))}\right)^{\frac{1}{p}}\left(f_{B(z, 2 \varepsilon)} Q^{\frac{1}{p-1}}(\zeta) d m(\zeta)\right)^{\frac{p-1}{p}}
$$

Consequently, almost everywhere we have

$$
L(z, f) \leq \limsup _{\varepsilon \rightarrow 0} \frac{d(f B(z, \varepsilon))}{\varepsilon} \leq \gamma_{p} \varphi^{\frac{1}{p}}(z) \cdot Q^{\frac{1}{p}}(z)<\infty .
$$

\section{$\S 5$. Finite Lipschitz PROPERTy}

In this section, we establish an analog of Gehring's theorem on the Lipschitz property for the lower $Q$-homeomorphisms relative to the $p$-module (see, e.g., [39, Theorem 2]).

Let $D$ be a domain in $\mathbb{C}$. We say that a homeomorphism $f: D \rightarrow \mathbb{C}$ is finite Lipschitzian if

$$
L(z, f)=\limsup _{\zeta \rightarrow z} \frac{|f(\zeta)-f(z)|}{|\zeta-z|}<\infty
$$

for all $z \in D$. Obviously, every Lipschitzian mapping is finite Lipschitzian.

The next lemma gives a condition ensuring the local Lipschitz property at a point for lower $Q$-homeomorphisms relative to the $p$-module with $p>2$. 
Lemma 8. Let $D$ and $D^{\prime}$ be domains in $\mathbb{C}$, let $Q: D \rightarrow[0, \infty]$ be a locally $L^{\frac{1}{p-1}}-$ function, and let $f: D \rightarrow D^{\prime}$ be a lower $Q$-homeomorphism relative to the $p$-module at a point $z_{0} \in D$ such that

$$
Q_{0}=\limsup _{\varepsilon \rightarrow 0}\left(f_{B\left(z_{0}, \varepsilon\right)} Q^{\frac{1}{p-1}}(\zeta) d m(\zeta)\right)^{p-1}<\infty .
$$

Then for $p>2$ we have

$$
L\left(z_{0}, f\right)=\limsup _{z \rightarrow z_{0}} \frac{\left|f(z)-f\left(z_{0}\right)\right|}{\left|z-z_{0}\right|} \leq \lambda_{p} Q_{0}^{\frac{1}{p-2}},
$$

where $\lambda_{p}$ is a positive constant depending only on $p$.

Proof. Consider the ring condenser

$$
\mathcal{E}=\left(B\left(z_{0}, r_{2}\right), \overline{B\left(z_{0}, r_{1}\right)}\right)
$$

with $0<r_{1}<r_{2}<d_{0}$, and the $\operatorname{ring} R=R\left(z_{0}, r_{1}, r_{2}\right)=\left\{\zeta \in \mathbb{C}: r_{1}<\left|\zeta-z_{0}\right|<r_{2}\right\}$.

Observe that the function

$$
\eta(t)= \begin{cases}\frac{1}{r_{2}-r_{1}}, & t \in\left(r_{1}, r_{2}\right), \\ 0, & t \in \mathbb{R} \backslash\left(r_{1}, r_{2}\right),\end{cases}
$$

satisfies condition (31). Then Lemma 6 implies the inequality

$$
\operatorname{cap}_{p^{\prime}} f \mathcal{E} \leq\left(r_{2}-r_{1}\right)^{-p^{\prime}} \int_{R\left(z_{0}, r_{1}, r_{2}\right)} Q^{\frac{1}{p-1}}(\zeta) d m(\zeta) .
$$

Next, choosing $r_{1}=2 \varepsilon$ and $r_{2}=4 \varepsilon$, we obtain

$$
\bigcap_{p^{\prime}}\left(f B\left(z_{0}, 4 \varepsilon\right), \overline{f B\left(z_{0}, 2 \varepsilon\right)}\right) \leq(2 \varepsilon)^{-p^{\prime}} \int_{B\left(z_{0}, 4 \varepsilon\right)} Q^{\frac{1}{p-1}}(\zeta) d m(\zeta) .
$$

On the other hand, by (9) we have

$$
\operatorname{cap}_{p^{\prime}}\left(f B\left(z_{0}, 4 \varepsilon\right), \overline{f B\left(z_{0}, 2 \varepsilon\right)}\right) \geq C_{p}\left[m\left(f B\left(z_{0}, 2 \varepsilon\right)\right)\right]^{\frac{p-2}{2(p-1)}},
$$

where $C_{p}$ is a positive constant depending only on $p$.

Combining (55) and (56), we obtain

$$
\frac{m\left(f B\left(z_{0}, 2 \varepsilon\right)\right)}{m\left(B\left(z_{0}, 2 \varepsilon\right)\right)} \leq c_{p}\left[f_{B\left(z_{0}, 4 \varepsilon\right)} Q^{\frac{1}{p-1}}(\zeta) d m(\zeta)\right]^{\frac{2(p-1)}{p-2}},
$$

where $c_{p}$ is a positive constant depending only on $p$.

Together with (54) (where we put $r_{1}=\varepsilon$ and $r_{2}=2 \varepsilon$ ), this gives the estimate

$$
\operatorname{cap}_{p^{\prime}}\left(f B\left(z_{0}, 2 \varepsilon\right), \overline{f B\left(z_{0}, \varepsilon\right)}\right) \leq \varepsilon^{-p^{\prime}} \int_{B\left(z_{0}, 2 \varepsilon\right)} Q^{\frac{1}{p-1}}(\zeta) d m(\zeta) .
$$

On the other hand, inequality (8) implies

$$
\operatorname{cap}_{p^{\prime}}\left(f B\left(z_{0}, 2 \varepsilon\right), \overline{f B\left(z_{0}, \varepsilon\right)}\right) \geq \widetilde{C}_{p}\left(\frac{d^{p}\left(\overline{f B\left(z_{0}, \varepsilon\right)}\right)}{m\left(f B\left(z_{0}, 2 \varepsilon\right)\right)}\right)^{\frac{1}{p-1}}
$$

where $\widetilde{C}_{p}$ is a positive constant depending only on $p$.

Combining (58) and (59), we find

$$
\frac{d\left(\overline{f B\left(z_{0}, \varepsilon\right)}\right)}{\varepsilon} \leq \gamma_{p}\left(\frac{m\left(f B\left(z_{0}, 2 \varepsilon\right)\right)}{m\left(B\left(z_{0}, 2 \varepsilon\right)\right)}\right)^{\frac{1}{p(p-1)}}\left(f_{B\left(z_{0}, 2 \varepsilon\right)} Q^{\left.\frac{1}{p-1}(\zeta) d m(\zeta)\right)^{\frac{p-1}{p}}},\right.
$$

where $\gamma_{p}$ is a positive constant depending only on $p$. 
Together with (57), this yields

$$
\frac{d\left(\overline{f B\left(z_{0}, \varepsilon\right)}\right)}{\varepsilon} \leq \lambda_{p}\left(f_{B\left(z_{0}, 4 \varepsilon\right)} Q^{\frac{1}{p-1}}(\zeta) d m(\zeta)\right)^{\frac{2(p-1)}{p(p-2)}}\left[f_{B\left(z_{0}, 2 \varepsilon\right)} Q^{\frac{1}{p-1}}(\zeta) d m(\zeta)\right]^{\frac{p-1}{p}} .
$$

Taking the upper limit as $\varepsilon \rightarrow 0$, we immediately get the claim:

$$
L\left(z_{0}, f\right)=\limsup _{z \rightarrow z_{0}} \frac{\left|f(z)-f\left(z_{0}\right)\right|}{\left|z-z_{0}\right|} \leq \limsup _{\varepsilon \rightarrow 0} \frac{d\left(\overline{f B\left(z_{0}, \varepsilon\right)}\right)}{\varepsilon} \leq \lambda_{p} Q_{0}^{\frac{1}{p-2}},
$$

where $\lambda_{p}$ is a positive constant depending only on $p$.

Theorem 2. Let $D$ and $D^{\prime}$ be domains in $\mathbb{C}$, let $Q: D \rightarrow[0, \infty]$ be a locally $L_{\frac{1}{p-1}}$-function, and let $f$ be a lower $Q$-homeomorphism relative to the $p$-module in $D$ such that

$$
\limsup _{\varepsilon \rightarrow 0} f_{B\left(z_{0}, \varepsilon\right)} Q^{\frac{1}{p-1}}(z) d m(z)<\infty \quad \text { for all } z_{0} \in D .
$$

Then for $p>2$ the homeomorphism $f$ is finite Lipschitzian.

Remark.. By [2, Lemma 10.6], the finite Lipchitzian mappings possess the $N$-property relative to the Hausdorf measures, and, thus, are absolutely continuous on curves.

Corollary 1. Let $D$ and $D^{\prime}$ be domains in $\mathbb{C}$, and let $f: D \rightarrow D^{\prime}$ be a lower $Q$-homeomorphism relative to the $p$-module in $D$ such that $Q(z) \leq K$ for a.e. $z \in D$. Then, for $p>2$, we have

$$
L\left(z_{0}, f\right)=\limsup _{z \rightarrow z_{0}} \frac{\left|f(z)-f\left(z_{0}\right)\right|}{\left|z-z_{0}\right|} \leq \lambda_{p} K^{\frac{1}{p-2}} \quad \text { for all } z_{0} \in D,
$$

where $\lambda_{p}$ is a positive constant depending only on $p$; in other words, the homeomorphism $f$ is locally Lipschitz.

\section{$\S 6$. HÖLDER PROPERTY FOR THE LOWER $Q$-HOMEOMORPHISMS RELATIVE TO THE $p$-MODULE}

Theorem 3. Let $D$ and $D^{\prime}$ be domains in $\mathbb{C}$, and let $f: D \rightarrow D^{\prime}$ be a lower $Q$-homeomorphism relative to the $p$-module in $D$ with $p>2$ and $Q(z) \in L_{s}\left(B_{0}\right)$, $B_{0}=B\left(z_{0}, \frac{1}{4} \operatorname{dist}\left(z_{0}, \partial D\right)\right), s>\frac{2}{p-2}$. Then

$$
\left|f(z)-f\left(z_{0}\right)\right| \leq \lambda_{p}\|Q\|_{s}^{\frac{1}{p-2}}\left|z-z_{0}\right|^{1-\frac{2}{s(p-2)}}
$$

for all $z \in B_{0}$, where $\|Q\|_{s}=\left(\int_{B_{0}} Q^{s}(\zeta) d m(\zeta)\right)^{\frac{1}{s}}$ is the norm in the space $L_{s}\left(B_{0}\right)$, and $\lambda_{p}$ is a positive constant depending only on $p$.

Proof. Consider the ring condenser

$$
\mathcal{E}=\left(B\left(z_{0}, r_{2}\right), \overline{B\left(z_{0}, r_{1}\right)}\right)
$$

with $0<r_{1}<r_{2}<d_{0}$, and the $\operatorname{ring} R=R\left(z_{0}, r_{1}, r_{2}\right)=\left\{\zeta \in \mathbb{C}: r_{1}<\left|\zeta-z_{0}\right|<r_{2}\right\}$. Observe that the function

$$
\eta(t)= \begin{cases}\frac{1}{r_{2}-r_{1}}, & t \in\left(r_{1}, r_{2}\right), \\ 0, & t \in \mathbb{R} \backslash\left(r_{1}, r_{2}\right),\end{cases}
$$

satisfies condition (31). Then, by Lemma 6.

$$
\operatorname{cap}_{p^{\prime}} f \mathcal{E} \leq\left(r_{2}-r_{1}\right)^{-p^{\prime}} \int_{R\left(z_{0}, r_{1}, r_{2}\right)} Q^{\frac{1}{p-1}}(\zeta) d m(\zeta)
$$


The Hölder inequality yields

$$
\operatorname{cap}_{p^{\prime}} f \mathcal{E} \leq \frac{\left(\pi r_{2}^{2}\right)^{\frac{s p-s-1}{s(p-1)}}}{\left(r_{2}-r_{1}\right)^{p^{\prime}}}\|Q\|_{s}^{\frac{1}{p-1}}
$$

where $\|Q\|_{s}=\left(\int_{B_{0}} Q^{s}(\zeta) d m(\zeta)\right)^{\frac{1}{s}}$.

Choosing $\varepsilon=\left|z-z_{0}\right|, r_{1}=2 \varepsilon$, and $r_{2}=4 \varepsilon$, we get

$$
\operatorname{cap}_{p^{\prime}}\left(f B\left(z_{0}, 4 \varepsilon\right), \overline{f B\left(z_{0}, 2 \varepsilon\right)}\right) \leq \gamma_{1}\|Q\|_{s}^{\frac{1}{p-1}} \cdot \varepsilon^{\frac{p s-2 s-2}{s(p-1)}},
$$

where $\gamma_{1}$ is a positive constant depending only on $p$.

On the other hand, inequality (9) implies

$$
\operatorname{cap}_{p^{\prime}}\left(f B\left(z_{0}, 4 \varepsilon\right), \overline{f B\left(z_{0}, 2 \varepsilon\right)}\right) \geq \gamma_{2}\left[m\left(\overline{f B\left(z_{0}, 2 \varepsilon\right)}\right)\right]^{\frac{p-2}{2(p-1)}},
$$

where $\gamma_{2}$ is a positive constant depending only on $p$.

Combining (65) and (66), we see that

$$
m\left(f B\left(z_{0}, 2 \varepsilon\right)\right) \leq \gamma_{3}\|Q\|_{s}^{\frac{2}{p-2}} \varepsilon^{\frac{2(p s-2 s-2)}{s(p-2)}},
$$

where $\gamma_{3}$ is a positive constant depending only on $p$.

Next, we choose $r_{1}=\varepsilon$ and $r_{2}=2 \varepsilon$ in (64), obtaining

$$
\operatorname{cap}_{p^{\prime}}\left(f B\left(z_{0}, 2 \varepsilon\right), \overline{f B\left(z_{0}, \varepsilon\right)}\right) \leq \gamma_{4}\|Q\|_{s}^{\frac{1}{p-1}} \cdot \varepsilon^{\frac{p s-2 s-2}{s(p-1)}} .
$$

On the other hand, from (8) it follows that

$$
\operatorname{cap}_{p^{\prime}}\left(f B\left(z_{0}, 2 \varepsilon\right), \overline{f B\left(z_{0}, \varepsilon\right)}\right) \geq\left(\gamma_{5} \frac{d^{p}\left(\overline{f B\left(z_{0}, \varepsilon\right)}\right)}{m\left(f B\left(z_{0}, 2 \varepsilon\right)\right)}\right)^{\frac{1}{p-1}}
$$

where $\gamma_{5}$ is a positive constant depending only on $p$.

Together, estimates (67)- 69) show that

$$
d\left(\overline{f B\left(z_{0}, \varepsilon\right)}\right) \leq \gamma_{p}\|Q\|_{s}^{\frac{1}{p-2}} \varepsilon^{1-\frac{2}{s(p-2)}},
$$

where $\gamma_{p}$ is a positive constant depending only on $p$. Now, estimate (62) follows from the last relation and the obvious inequality

$$
d\left(\overline{f B\left(z_{0}, \varepsilon\right)}\right) \geq\left|f(z)-f\left(z_{0}\right)\right| .
$$

\section{§7. On the logarithmic Hölder PROPERTy}

Lemma 9. Let $D$ and $D^{\prime}$ be domains in $\mathbb{C}$, and let $f: D \rightarrow D^{\prime}$ be a lower $Q$-homeomorphism relative to the $p$-module in $D$ for $p>2$ such that

$$
\int_{R\left(z_{0}, r_{1}, r_{2}\right)} \frac{Q^{\frac{1}{p-1}}(\zeta) d m(\zeta)}{\left|\zeta-z_{0}\right|^{p^{\prime}}} \leq C_{z_{0}} \cdot \ln ^{\kappa}\left(\frac{r_{2}}{r_{1}}\right), \quad \kappa<p^{\prime},
$$

for any $0<r_{1}<r_{2}<\operatorname{dist}\left(z_{0}, \partial D\right)$. Then for $p>2$ we have

$$
\operatorname{cap}_{p^{\prime}}\left(f B\left(z_{0}, r_{2}\right), \overline{f B\left(z_{0}, r_{1}\right)}\right) \leq C_{z_{0}} \cdot \ln ^{\kappa-p^{\prime}}\left(\frac{r_{2}}{r_{1}}\right) .
$$

Proof. Consider the ring condenser

$$
\mathcal{E}=\left(B\left(z_{0}, r_{2}\right), \overline{B\left(z_{0}, r_{1}\right)}\right)
$$

with $0<r_{1}<r_{2}<d_{0}$ and the ring $R=R\left(z_{0}, r_{1}, r_{2}\right)=\left\{\zeta \in \mathbb{C}: r_{1}<\left|\zeta-z_{0}\right|<r_{2}\right\}$.

Observe tht the function

$$
\eta(t)= \begin{cases}\frac{1}{t \ln \left(\frac{r_{2}}{r_{1}}\right)}, & t \in\left(r_{1}, r_{2}\right), \\ 0, & t \in \mathbb{R} \backslash\left(r_{1}, r_{2}\right),\end{cases}
$$


satisfies condition (31). Then, by Lemma 6.

$$
\operatorname{cap}_{p^{\prime}} f \mathcal{E} \leq \ln ^{-p^{\prime}}\left(\frac{r_{2}}{r_{1}}\right) \int_{R\left(z_{0}, r_{1}, r_{2}\right)} \frac{Q^{\frac{1}{p-1}}(\zeta) d m(\zeta)}{\left|z-z_{0}\right|^{p^{\prime}}} .
$$

Recalling condition (70), we arrive at (71).

Theorem 4. Let $D$ and $D^{\prime}$ be domains in $\mathbb{C}$, and let $f: D \rightarrow D^{\prime}$ be a lower $Q$-homeomorphism relative to the $p$-module for $p>2$ such that

$$
\int_{R\left(z_{0}, \varepsilon_{1}, \varepsilon_{2}\right)} \frac{Q^{\frac{1}{p-1}}(\zeta) d m(\zeta)}{\left|\zeta-z_{0}\right|^{p^{\prime}}} \leq C_{z_{0}} \cdot \ln ^{\kappa}\left(\frac{\varepsilon_{2}}{\varepsilon_{1}}\right), \quad \kappa<p^{\prime},
$$

for any $0<\varepsilon_{1}<\varepsilon_{2}<\operatorname{dist}\left(z_{0}, \partial D\right)$. Then

$$
m\left(f B\left(z_{0}, \varepsilon\right)\right) \leq \lambda_{0} C_{z_{0}}^{\frac{2(p-1)}{p-2}} \ln ^{-\frac{2(\kappa+p-\kappa p)}{p-2}}\left(\frac{1}{\varepsilon}\right)
$$

for all $\varepsilon<\delta_{0} \leq \min \left\{1, \operatorname{dist}^{2}\left(z_{0}, \partial D\right)\right\}$, where $\lambda_{0}=\lambda(p, \kappa)$ is a positive constant depending only on $p$ and $\kappa$.

Proof. Consider the condenser

$$
\mathcal{E}_{\varepsilon}=\left(B\left(x_{0}, \sqrt{\varepsilon}\right), \overline{B\left(x_{0}, \varepsilon\right)}\right) .
$$

By Lemma 9, we have

$$
\operatorname{cap}_{p^{\prime}} f \mathcal{E}_{\varepsilon} \leq 2^{p-\kappa} C_{z_{0}} \cdot \ln ^{\kappa-p}\left(\frac{1}{\varepsilon}\right)
$$

Using (9), we see that

$$
\operatorname{cap}_{p^{\prime}} f \mathcal{E}_{\varepsilon} \geq \gamma_{p}\left[m\left(\overline{f B\left(z_{0}, \varepsilon\right)}\right)\right]^{\frac{p-2}{2(p-1)}},
$$

where $\gamma_{p}$ is a constant depending only on $p$. Combining (75) and (76), we get the inequality

$$
m\left(f B\left(z_{0}, \varepsilon\right)\right) \leq \lambda_{0} C_{z_{0}}^{\frac{2(p-1)}{p-2}} \ln ^{-\frac{2(\kappa+p-\kappa p)}{p-2}}\left(\frac{1}{\varepsilon}\right),
$$

where $\lambda_{0}=\lambda(p, \kappa)$ is a positive constant depending only on $p$ and $\kappa$.

Theorem 5. Let $D$ and $D^{\prime}$ be domains in $\mathbb{C}$, and let $f: D \rightarrow D^{\prime}$ be a lower $Q$-homeomorphism relative to the $p$-module in $D$ for $p>2$ such that

$$
\int_{R\left(z_{0}, \varepsilon_{1}, \varepsilon_{2}\right)} \frac{Q^{\frac{1}{p-1}}(\zeta) d m(\zeta)}{\left|\zeta-z_{0}\right|^{p^{\prime}}} \leq C_{z_{0}} \cdot \ln ^{\kappa}\left(\frac{\varepsilon_{2}}{\varepsilon_{1}}\right), \quad \kappa<p^{\prime},
$$

for any $0<\varepsilon_{1}<\varepsilon_{2}<\operatorname{dist}\left(z_{0}, \partial D\right)$. Then

$$
\left|f(z)-f\left(z_{0}\right)\right| \leq \lambda_{0} C_{z_{0}}^{\frac{p-1}{p-2}} \ln ^{-\frac{\kappa+p-\kappa p}{p-2}} \frac{1}{\left|z-z_{0}\right|}
$$

for any $z \in B\left(z_{0}, \delta_{0}\right)$, where $\delta_{0} \leq \min \left\{1\right.$, $\left.\operatorname{dist}^{4}\left(z_{0}, \partial D\right)\right\}$ and $\lambda_{0}=\lambda(p, \kappa)$ is a positive constant depending on $p$ and $\kappa$.

Proof. We put $\varepsilon=\left|z-z_{0}\right|<\delta_{0}$. Consider the condenser

$$
\mathcal{E}_{\varepsilon}=\left(B\left(z_{0}, \sqrt{\varepsilon}\right), \overline{B\left(z_{0}, \varepsilon\right)}\right) .
$$

By (71), we have

$$
\operatorname{cap}_{p^{\prime}} f \mathcal{E}_{\varepsilon} \leq 2^{p^{\prime}-\kappa} C_{z_{0}} \cdot \ln ^{\kappa-p^{\prime}}\left(\frac{1}{\varepsilon}\right)
$$


On the other hand, inequality (8) implies

$$
\operatorname{cap}_{p^{\prime}} f \mathcal{E}_{\varepsilon} \geq C_{p}\left(\frac{d^{p}\left(\overline{f B\left(z_{0}, \varepsilon\right)}\right)}{m\left(f B\left(z_{0}, \sqrt{\varepsilon}\right)\right)}\right)^{\frac{1}{p-1}}
$$

where $C_{p}$ is a positive constant depending only on $p$.

By (80) and (81), we have

$$
d\left(\overline{f B\left(z_{0}, \varepsilon\right)}\right) \leq \lambda_{p, \kappa} \cdot C_{z_{0}}^{\frac{p-1}{p}} \cdot \ln ^{-\frac{\kappa+p-\kappa p}{p}}\left(\frac{1}{\varepsilon}\right) m^{\frac{1}{p}}\left(f B\left(z_{0}, \sqrt{\varepsilon}\right)\right) .
$$

Theorem 4 yields an estimate for the area of the image of the disk $B\left(z_{0}, \sqrt{\varepsilon}\right)$ :

$$
m\left(f B\left(z_{0}, \sqrt{\varepsilon}\right)\right) \leq \gamma_{0} C_{z_{0}}^{\frac{2(p-1)}{p-2}} \ln ^{-\frac{2(\kappa+p-\kappa p)}{p-2}}\left(\frac{1}{\varepsilon}\right) .
$$

Finally, combining (82) and (83), we get

$$
\left|f(z)-f\left(z_{0}\right)\right| \leq d\left(\overline{f B\left(z_{0}, \varepsilon\right)}\right) \leq \lambda_{0} C_{z_{0}}^{\frac{p-1}{p-2}} \ln ^{-\frac{\kappa+p-\kappa p}{p-2}} \frac{1}{\varepsilon} .
$$

Corollary 2. Let $D$ and $D^{\prime}$ be domains in $\mathbb{C}$, and let $f: D \rightarrow D^{\prime}$ be a lower $Q$-homeomorphism relative to the $p$-module for $p>0$, with $Q(z) \in L_{\frac{2}{p-2}}\left(B\left(z_{0}, \delta_{0}\right)\right)$. Then

$$
\left|f(z)-f\left(z_{0}\right)\right| \leq \lambda_{p}\|Q\|_{\frac{2}{p-2}}^{\frac{1}{p-1}} \ln ^{-\frac{p}{2(p-2)}} \frac{1}{\left|z-z_{0}\right|}
$$

for all $z \in B\left(z_{0}, \delta_{0}\right)$, where $\delta_{0} \leq \min \left\{1, \operatorname{dist}^{4}\left(z_{0}, \partial D\right)\right\}$,

$$
\|Q\|_{\frac{2}{p-2}}=\left(\int_{B\left(z_{0}, \delta_{0}\right)} Q^{\frac{2}{p-2}}(\zeta) d m(\zeta)\right)^{\frac{p-2}{2}}
$$

is the norm in the space $L_{\frac{2}{p-2}}\left(B\left(z_{0}, \delta_{0}\right)\right)$, and $\lambda_{p}$ is a positive constant depending only on $p$.

Proof. Indeed, the Hölder inequality yields

$$
\begin{aligned}
& \int_{R\left(z_{0}, \varepsilon_{1}, \varepsilon_{2}\right)} \frac{Q^{\frac{1}{p-1}}(\zeta) d m(\zeta)}{\left|\zeta-z_{0}\right|^{p^{\prime}}} \\
& \quad \leq\left(\int_{R\left(z_{0}, \varepsilon_{1}, \varepsilon_{2}\right)} Q^{\frac{2}{p-2}}(\zeta) d m(\zeta)\right)^{\frac{p-2}{2(p-1)}}\left(\int_{R\left(z_{0}, \varepsilon_{1}, \varepsilon_{2}\right)} \frac{d m(\zeta)}{\left|\zeta-z_{0}\right|^{2}}\right)^{\frac{p}{2(p-1)}} .
\end{aligned}
$$

Consequently,

$$
\int_{R\left(z_{0}, \varepsilon_{1}, \varepsilon_{2}\right)} \frac{Q^{\frac{1}{p-1}}(\zeta) d m(\zeta)}{\left|\zeta-z_{0}\right|^{p}} \leq\left(\int_{B\left(z_{0}, \delta_{0}\right)} Q^{\frac{2}{p-2}}(\zeta) d m(\zeta)\right)^{\frac{p-2}{2(p-1)}}\left(2 \pi \ln \left(\frac{\varepsilon_{2}}{\varepsilon_{1}}\right)\right)^{\frac{p}{2(p-1)}} .
$$

Now, to get (84) it suffices to apply Theorem 5 with $\kappa=\frac{p}{2(p-1)}$ and

$$
C_{z_{0}}=(2 \pi)^{\frac{p}{2(p-1)}}\|Q\|_{\frac{2}{p-2}}^{\frac{1}{p-1}} .
$$

Theorem 6. Let $D$ and $D^{\prime}$ be domains in $\mathbb{C}$, and let $f: D \rightarrow D^{\prime}$ be a lower $Q$-homeomorphism relative to the $p$-module for $p>2$, with

$$
\|Q\|_{\frac{1}{p-1}}(r) \leq Q_{z_{0}} r \text { for a.e. } r \in\left(0, \delta_{0}\right) .
$$

Then

$$
\left|f(z)-f\left(z_{0}\right)\right| \leq \lambda_{p} Q_{z_{0}}^{\frac{1}{p-2}} \ln ^{-\frac{1}{p-2}} \frac{1}{\left|z-z_{0}\right|}
$$


for all $z \in B\left(z_{0}, \delta_{0}\right)$, where $\delta_{0} \leq \min \left\{1\right.$, $\left.\operatorname{dist}^{4}\left(z_{0}, \partial D\right)\right\}$ and $\lambda_{p}$ is a positive constant depending only on $p$.

Proof. Indeed, using condition (86) and the Fubini theorem, we get

$$
\int_{R\left(z_{0}, \varepsilon_{1}, \varepsilon_{2}\right)} \frac{Q^{\frac{1}{p-1}}(\zeta) d m(\zeta)}{\left|\zeta-z_{0}\right|^{p^{\prime}}}=\int_{\varepsilon_{1}}^{\varepsilon_{2}} r^{-p^{\prime}}\|Q\|_{\frac{1}{p-1}}^{p-1}(r) d r \leq Q_{z_{0}}^{p-1} \ln \frac{\varepsilon_{1}}{\varepsilon_{2}} .
$$

\section{§8. Area distortion for a Disk}

In this section we obtain sharp estimates for the area of the image of a disk under lower $Q$-homeomorphisms relative to the $p$-module. For the first time, for quasiconformal mappings, such an estimate was presented in the book [1] by M. A. Lavrent'ev. In the sequel we put $B_{r}=B(0, r)=\{z \in \mathbb{C}:|z|<r\}$ and $\mathbb{B}=B(0,1)=\{z \in \mathbb{C}:|z|<1\}$.

Theorem 7. Let $f: \mathbb{B} \rightarrow \mathbb{B}$ be a lower $Q$-homeomorphism relative to the p-module. Then, for $p>2$ we have

$$
m\left(f B_{r}\right) \leq \pi \cdot\left(1+(2 \pi)^{p-1}(p-2) \int_{r}^{1} \frac{d t}{\|Q\|_{\frac{1}{p-1}}(t)}\right)^{\frac{2}{2-p}}
$$

and for $p=2$ we have

$$
m\left(f B_{r}\right) \leq \pi \exp \left\{-4 \pi \int_{r}^{1} \frac{d t}{\|Q\|_{1}(t)}\right\} .
$$

Proof. Consider the ring $R_{t}=\{z \in \mathbb{B}: t<|z|<t+\triangle t\}$ and the condenser $\left(A_{t+\triangle t}, C_{t}\right)$, where $C_{t}=\bar{B}_{t}, A_{t+\Delta t}=B_{t+\Delta t}$. Then $\left(f A_{t+\Delta t}, f C_{t}\right)$ is a ring condenser in $\mathbb{C}$, and by (7) we have

$$
\operatorname{cap}_{p^{\prime}}\left(f A_{t+\triangle t}, f C_{t}\right)=\mathcal{M}_{p^{\prime}}\left(\triangle\left(\partial f A_{t+\triangle t}, \partial f C_{t} ; f R_{t}\right)\right) .
$$

Inequality (10) implies the estimate

$$
\operatorname{cap}_{p^{\prime}}\left(f A_{t+\Delta t}, f C_{t}\right) \geq \frac{[\inf l(\sigma)]^{\frac{p}{p-1}}}{m\left[f A_{t+\Delta t} \backslash f C_{t}\right]^{\frac{1}{p-1}}},
$$

where $l(\sigma)$ is the arc length, $\sigma$ is a smooth (infinitely differentiable) curve that is the boundary $\sigma=\partial U$ of a bounded open set $U$ containing $f C_{t}$ and contained, together with its closure $\bar{U}$, in $f A_{t+\triangle t}$; the infimum is taken over all such curves $\sigma$.

On the other hand, by Lemma 3 ,

$$
\operatorname{cap}_{p^{\prime}}\left(f A_{t+\triangle t}, f C_{t}\right) \leq\left(\int_{t}^{t+\triangle t} \frac{d r}{\|Q\|_{\frac{1}{p-1}}(r)}\right)^{-\frac{1}{p-1}} .
$$

Combining (91) and (92), we get

$$
\frac{[\inf l(\sigma)]^{\frac{p}{p-1}}}{m\left[f A_{t+\Delta t} \backslash f C_{t}\right]^{\frac{1}{p-1}}} \leq\left(\int_{t}^{t+\Delta t} \frac{d r}{\|Q\|_{\frac{1}{p-1}}(r)}\right)^{-\frac{1}{p-1}} .
$$

Next we use the isoperimetrical inequality

$$
\inf l(\sigma) \geq 2 \cdot \pi^{\frac{1}{2}}\left(m\left(f C_{t}\right)\right)^{\frac{1}{2}}
$$

to show that

$$
2 \cdot \pi^{\frac{1}{2}}\left(m\left(f C_{t}\right)\right)^{\frac{1}{2}} \leq\left(\frac{m\left(f A_{t+\Delta t} \backslash f C_{t}\right)}{\int_{t}^{t+\triangle t} \frac{d r}{\|Q\|_{\frac{1}{p-1}}(r)}}\right)^{\frac{1}{p}}
$$


Putting $\Phi(t):=m\left(f B_{t}\right)$, from (95) we deduce the estimate

$$
2 \cdot \pi^{\frac{1}{2}} \Phi^{\frac{1}{2}}(t) \leq\left(\frac{\frac{\Phi(t+\Delta t)-\Phi(t)}{\Delta t}}{\frac{1}{\Delta t} \int_{t}^{t+\Delta t} \frac{d r}{\|Q\|_{\frac{1}{p-1}}(r)}}\right)^{\frac{1}{p}} .
$$

Observe that, since $f$ is a homeomorphism, by Lemma 3 we have

$$
\|Q\|_{\frac{1}{p-1}}^{-1}(r) \in L_{\mathrm{loc}}^{1}(0,1) \text {. }
$$

Now we pass to the limit in (96) as $\Delta t \rightarrow 0$. Since the function $\Phi(t)$ is monotone increasing with respect to $t \in(0,1)$, we see that the derivative $\Phi^{\prime}(t)$ exists for a.e. $t$, and that

$$
2^{p} \pi^{\frac{p}{2}}\|Q\|_{\frac{1}{p-1}}^{-1}(t) \leq \frac{\Phi^{\prime}(t)}{\Phi^{\frac{p}{2}}(t)} .
$$

Consider inequality (97) for $p>2$. Integrating the two sides of (97) over $t \in[r, 1]$ and using the estimate

$$
\int_{r}^{1} \frac{\Phi^{\prime}(t)}{\Phi^{\frac{p}{2}}(t)} d t \leq \frac{2}{2-p}\left(\Phi^{\frac{2-p}{2}}(1)-\Phi^{\frac{2-p}{2}}(r)\right)
$$

(see, e.g., [32, Theorem IV.7.4]), we obtain the inequality

$$
2^{p} \pi^{\frac{p}{2}} \int_{r}^{1} \frac{d t}{\|Q\|_{\frac{1}{p-1}}(t)} \leq \frac{2}{2-p}\left(\Phi^{\frac{2-p}{2}}(1)-\Phi^{\frac{2-p}{2}}(r)\right),
$$

which implies

$$
\Phi(r) \leq\left(\Phi^{\frac{2-p}{2}}(1)-2^{p-1}(2-p) \pi^{\frac{p}{2}} \int_{r}^{1} \frac{d t}{\|Q\|_{\frac{1}{p-1}}(t)}\right)^{\frac{2}{2-p}} .
$$

Finally, recalling that $m(f \mathbb{B}) \leq \pi$, we arrive at (88).

It remains to treat the case of $p=2$. Then (97) takes the form

$$
\frac{4 \pi}{\|Q\|_{1}(t)} \leq \frac{\Phi^{\prime}(t)}{\Phi(t)}
$$

Integrating the two sides in (101) over $t \in[r, 1]$ and using the estimate

$$
\int_{r}^{1} \frac{\Phi^{\prime}(t)}{\Phi(t)} d t \leq \ln \frac{\Phi(1)}{\Phi(r)}
$$

(see, e.g., [32, Theorem IV.7.4]), we obtain

$$
4 \pi \int_{r}^{1} \frac{d t}{\|Q\|_{1}(t)} \leq \ln \frac{\Phi(1)}{\Phi(r)} .
$$

Consequently,

$$
\exp \left\{4 \pi \int_{r}^{1} \frac{d t}{\|Q\|_{1}(t)}\right\} \leq \frac{\Phi(1)}{\Phi(r)}
$$

whence

$$
\Phi(r) \leq \Phi(1) \cdot \exp \left\{-4 \pi \int_{r}^{1} \frac{d t}{\|Q\|_{1}(t)}\right\},
$$

which results in (89), because $\Phi(1) \leq \pi$.

Remark 1. Examples show that estimates (88) and (89) are both sharp. 


\section{§9. BEHAVIOR AT A POINT}

The theorem in the preceding section allows us to describe the asymptotic behavior of the lower $Q$-homeomorphisms relative to the $p$-module at a point.

Theorem 8. Let $f: \mathbb{B} \rightarrow \mathbb{B}$ be a lower $Q$-homeomorphism relative the p-module such that $f(0)=0$. Then, for $p>2$ we have

$$
\liminf _{z \rightarrow 0}|f(z)| \cdot\left(1+(2 \pi)^{p-1}(p-2) \int_{|z|}^{1} \frac{d t}{\|Q\|_{\frac{1}{p-1}}(t)}\right)^{\frac{1}{p-2}} \leq 1,
$$

and for $p=2$ we have

$$
\liminf _{z \rightarrow 0}|f(z)| \cdot \exp \left\{2 \pi \int_{|z|}^{1} \frac{d t}{\|Q\|_{1}(t)}\right\} \leq 1 .
$$

Proof. Put $l_{f}(r)=\min _{|z|=r}|f(z)|$. Since $f(0)=0$, we have $\pi l_{f}^{2}(r) \leq m\left(f B_{r}\right)$, whence

$$
l_{f}(r) \leq\left(\frac{m\left(f B_{r}\right)}{\pi}\right)^{\frac{1}{2}} .
$$

Thus, using (88) and (89), we see that

$$
\liminf _{z \rightarrow 0} \frac{|f(z)|}{\mathcal{R}_{p}(|z|)}=\liminf _{r \rightarrow 0} \frac{l_{f}(r)}{\mathcal{R}_{p}(r)} \leq \liminf _{r \rightarrow 0}\left(\frac{m\left(f B_{r}\right)}{\pi}\right)^{\frac{1}{2}} \cdot \frac{1}{\mathcal{R}_{p}(r)} \leq 1,
$$

where

$$
\mathcal{R}_{p}(r)=\left(1+(2 \pi)^{p-1}(p-2) \int_{r}^{1} \frac{d t}{\|Q\|_{\frac{1}{p-1}}(t)}\right)^{-\frac{1}{p-2}}
$$

if $p>2$, and

$$
\mathcal{R}_{p}(r)=\exp \left\{-2 \pi \int_{r}^{1} \frac{d t}{\|Q\|_{1}(t)}\right\}
$$

of $p=2$. The theorem is proved.

Corollary 3. Let $f: \mathbb{B} \rightarrow \mathbb{B}$ be a lower $Q$-homeomorphism relative to the $p$-module such that $f(0)=0$. Then for $p>2$ we have

$$
\liminf _{z \rightarrow 0}|f(z)| \cdot\left(\int_{|z|}^{1} \frac{d t}{\|Q\|_{\frac{1}{p-1}}(t)}\right)^{\frac{1}{p-2}} \leq(2 \pi)^{1-p}(p-2)^{\frac{1}{2-p}} .
$$

\section{§10. On the Relationship With the SoboleV Classes}

Let $D$ be a domain in $\mathbb{C}$. We recall that a mapping $f: D \rightarrow \mathbb{C}$ is said to have finite distortion if $f \in W_{\mathrm{loc}}^{1,1}$ and

$$
\left\|f^{\prime}(z)\right\|^{2} \leq K(z) \cdot J_{f}(z)
$$

for some a.e. finite function $K(z)$. For the first time, finite distortion mappings arose in the plane case for $f \in W_{\text {loc }}^{1,2}$ in the paper [41. Later, this condition was replaced by the requirement $f \in W_{\text {loc }}^{1,1}$, under the additional assumption that $J_{f} \in L_{\text {loc }}^{1}$ (see, e.g., the book [40]).

Let $p \in(1, \infty)$. We put

$$
K_{p, f}(z)= \begin{cases}\frac{\left\|f^{\prime}(z)\right\|^{p}}{J(z, f)} & \text { if } J(z, f) \neq 0 \\ 1 & \text { if } f^{\prime}(z)=0 \\ \infty & \text { otherwise. }\end{cases}
$$


Theorem 9. Let $D$ and $D^{\prime}$ be domains in $\mathbb{C}$, and let $f: D \rightarrow D^{\prime}$ be a homeomorphism with finite distortion. Then $f$ is a lower $Q$-homeomorphism relative to the p-module at every point $z_{0} \in D$ with $Q(z)=K_{p, f}(z)$.

Proof. Let $\mathfrak{B}$ denote the (Borel!) set of all points $z$ in $D$ at which $f$ has full differential with $J_{f}(z) \neq 0$. It is known that $\mathfrak{B}$ can be presented as the union of a countable collection of Borel sets $\mathfrak{B}_{l}, l=1,2, \ldots$, such that $f_{l}=\left.f\right|_{\mathfrak{B}_{l}}$ are bi-Lipschitz homeomorphisms (see, e.g., [37, Lemma 3.2.2]). There is no loss of generality in assuming that the $\mathfrak{B}_{l}$ are mutually disjoint. Let $\mathfrak{B}_{*}$ be the set of all points $z \in D$ where $f$ has full differential with $f_{z}=0=f_{\bar{z}}$.

Note that, by the well-known Gehring-Lehto-Men'shov theorem, the set $\mathfrak{B}_{0}=$ $D \backslash\left(\mathfrak{B} \cup \mathfrak{B}_{*}\right.$ ) has zero Lebesgue measure in $\mathbb{C}$ (see [38] and 44]). Consequently, by [42, Theorem 2.11] (see also [2, Lemma 9.1]), we have $l\left(\gamma \cap \mathfrak{B}_{0}\right)=0$ for a.e. dashed line $\gamma$ in $D$. We show also that $l\left(f(\gamma) \cap f\left(\mathfrak{B}_{0}\right)\right)=0$ for a.e. circle $\gamma$ with center at $z_{0}$.

This follows from the absolute continuity of $f$ on the closed subarcs $\gamma \cap D$ for a.e. circle $\gamma$. Indeed, the class $W_{\text {loc }}^{1,1}$ is invariant under the locally quasiisometric transformations of the independent variable (see, e.g., [31, Theorem 1.1.7]), and the functions in $W_{\text {loc }}^{1,1}$ are absolutely continuous on lines (see, e.g., [31, Theorem 1.1.3]). Applying, for example, the coordinate transformation $\log \left(z-z_{0}\right)$, we establish absolute continuity on a.e. circle $\gamma$ centered at $z_{0}$.

Thus, $l\left(\gamma_{*} \cap f\left(\mathfrak{B}_{0}\right)\right)=0$, where $\gamma_{*}=f(\gamma)$, for a.e. circle $\gamma$ centered at $z_{0}$. Now, let $\varrho_{*} \in \operatorname{adm} f(\Gamma), \varrho_{*} \equiv 0$ outside $f(D)$, where $\Gamma$ is the collection of the dashed lines formed by intersections with $D$ of all circles centered at $z_{0}$. Let $\varrho \equiv 0$ outside $D$, and let

$$
\varrho(z):=\varrho_{*}(f(z))\left(\left|f_{z}\right|+\left|f_{\bar{z}}\right|\right) \text { for a.e. } z \in D .
$$

Arguing piecewise on $\mathfrak{B}_{l}$ and using [37, Theorem 3.2.5] (for $m=1$ ), we see that

$$
\int_{\gamma} \varrho d s \geq \int_{\gamma_{*}} \varrho_{*} d s_{*} \geq 1 \text { for a.e. } \gamma \in \Gamma
$$

because $l\left(f(\gamma) \cap f\left(\mathfrak{B}_{0}\right)\right)=0$, and also $l\left(f(\gamma) \cap f\left(\mathfrak{B}_{*}\right)\right)=0$ for $p$-a.e. $\gamma \in \Gamma$ because $f$ is absolutely continuous on $p$-a.e. $\gamma \in \Gamma$. Consequently, $\varrho \in \operatorname{ext}_{p} \operatorname{adm} \Gamma$.

On the other hand, again arguing piecewise on $\mathfrak{B}_{l}$, we obtain the inequality

$$
\int_{D} \frac{\varrho^{p}(x)}{K_{p, f}(z)} d m(z) \leq \int_{f(D)} \varrho_{*}^{p}(w) d m(w)
$$

because $\varrho(z)=0$ on $\mathfrak{B}_{*}$. Therefore,

$$
M_{p}(f \Gamma) \geq \inf _{\varrho \in \operatorname{ext}_{p} \operatorname{adm} \Gamma} \int_{D} \frac{\varrho^{p}(z)}{K_{p, f}(z)} d m(z),
$$

i.e., indeed, $f$ is a lower $Q$-homeomorphism with $Q(z)=K_{p, f}(z)$ relative to the $p$-module.

Lemma 10. Let $D$ and $D^{\prime}$ be domains in $\mathbb{C}$, and let $f: D \rightarrow D^{\prime}$ be a finite distortion homeomorphism such that

$$
k_{p}\left(z_{0}\right)=\limsup _{\varepsilon \rightarrow 0}\left(f_{B\left(z_{0}, \varepsilon\right)} K_{p, f}^{\frac{1}{p-1}}(z) d m(z)\right)^{p-1}<\infty, \quad z_{0} \in D .
$$

Then for $p>2$ we have

$$
L\left(z_{0}, f\right)=\limsup _{z \rightarrow z_{0}} \frac{\left|f(z)-f\left(z_{0}\right)\right|}{\left|z-z_{0}\right|} \leq \lambda_{p} k_{p}^{\frac{1}{p-2}}\left(z_{0}\right),
$$

where $\lambda_{p}$ is a positive constant depending only on $p$. 
Theorem 10. Let $D$ and $D^{\prime}$ be domains in $\mathbb{C}$, and let $f: D \rightarrow D^{\prime}$ be a finite distortion homeomorphism such that

$$
\limsup _{\varepsilon \rightarrow 0}\left(f_{B\left(z_{0}, \varepsilon\right)} K_{p, f}^{\frac{1}{p-1}}(z) d m(z)\right)^{p-1}<\infty \quad \text { for all } z_{0} \in D .
$$

Then, for $p>2$, the homeomorphism $f$ is finite Lipschitzian.

Example 1. Let $p>2$, and let $f: \mathbb{B} \rightarrow \mathbb{B}$ be defined by

$$
f(z)=\frac{z}{|z|}\left(1+(p-2) \int_{|z|}^{1} \frac{d t}{t^{p-1} \ln ^{p-1}\left(\frac{e}{t}\right)}\right)^{\frac{1}{2-p}}
$$

for $z \neq 0$, with $f(0)=0$.

It is easy to check that $f$ belongs to $W_{\text {loc }}^{1,1}$ and is a finite distortion mapping. The tangent and radial dilations of $f$ are calculated by the formulas

$$
\delta_{T}=\frac{|f(z)|}{|z|}=\frac{\left(1+(p-2) \int_{|z|}^{1} \frac{d t}{t^{p-1} \ln ^{p-1}\left(\frac{e}{t}\right)}\right)^{\frac{1}{2-p}}}{|z|}
$$

and

$$
\delta_{r}=\frac{\partial|f(z)|}{\partial|z|}=\frac{\left(1+(p-2) \int_{|z|}^{1} \frac{d t}{t^{p-1} \ln ^{p-1}\left(\frac{e}{t}\right)}\right)^{\frac{p-1}{2-p}}}{\left(|z| \ln \frac{e}{|z|}\right)^{p-1}} .
$$

It is easily seen that $\delta_{T}>\delta_{r}$ and $\delta_{T}^{p-1}=\left(\ln \frac{e}{|z|}\right)^{p-1} \delta_{r}$. Consequently,

Observe that

$$
K_{p, f}(z)=\frac{\delta_{T}^{p}}{\delta_{T} \delta_{r}}=\frac{\delta_{T}^{p-1}}{\delta_{r}}=\left(\ln \frac{e}{|z|}\right)^{p-1} .
$$

$$
\limsup _{\varepsilon \rightarrow 0} f_{B(\varepsilon)} K_{p, f}^{\frac{1}{p-1}}(z) d m(z)=\infty .
$$

Nevertheless, the L'Hôpital rule shows that $\frac{|f(z)|}{|z|} \rightarrow \infty$ as $z \rightarrow 0$, i.e., the homeomorphism $f$ is not Lipschitz at zero.

Theorem 11. Let $D$ and $D^{\prime}$ be domains in $\mathbb{C}$, and let $f: D \rightarrow D^{\prime}$ be a finite distortion homeomorphism such that

$$
\left\|K_{p, f}(z)\right\|_{\frac{1}{p-1}}(r) \leq Q_{z_{0}} r \text { for a.e. } r \in\left(\varepsilon_{1}, \varepsilon_{2}\right) .
$$

Then

$$
\left|f(z)-f\left(z_{0}\right)\right| \leq \lambda_{p} Q_{z_{0}}^{\frac{1}{p-2}} \ln ^{-\frac{1}{p-2}} \frac{1}{\left|z-z_{0}\right|}
$$

for all $z \in B\left(z_{0}, \delta_{0}\right)$, where $\delta_{0} \leq \min \left\{1\right.$, $\left.\operatorname{dist}^{4}\left(z_{0}, \partial D\right)\right\}$ and $\lambda_{p}$ is a positive constant depending only on $p$.

Example 2. Let $p>2$, and let $f: \mathbb{B} \rightarrow \mathbb{B}$ be defined by the formula

$$
f(z)=\frac{z}{|z|}\left(1+(p-2) \ln \frac{1}{|z|}\right)^{\frac{1}{2-p}}
$$

for $z \neq 0$, with $f(0)=0$.

The tangent and radial dilations of $f$ can easily be computed:

$$
\delta_{T}=\frac{|f(z)|}{|z|}=\frac{\left(1+(p-2) \ln \frac{1}{|z|}\right)^{\frac{1}{2-p}}}{|z|}
$$


and

$$
\delta_{r}=\frac{\partial|f(z)|}{\partial|z|}=\frac{\left(1+(p-2) \ln \frac{1}{|z|}\right)^{\frac{p-1}{2-p}}}{|z|} .
$$

Observe that $\delta_{T}>\delta_{r}$ and $\delta_{T}^{p-1}=|z|^{2-p} \delta_{r}$. Thus,

$$
K_{p, f}(z)=\frac{\delta_{T}^{p}}{\delta_{T} \delta_{r}}=\frac{\delta_{T}^{p-1}}{\delta_{r}}=|z|^{2-p} .
$$

Consequently,

$$
\left\|K_{p, f}(z)\right\|_{\frac{1}{p-1}}(r)=\left(\int_{|z|=r} K_{p, f}^{\frac{1}{p-1}}(z)|d z|\right)^{p-1}=(2 \pi)^{p-1} r
$$

which leads to the estimate

$$
|f(z)| \leq(p-2)^{2-p} \ln ^{-\frac{1}{p-2}} \frac{1}{|z|} .
$$

\section{§11. Application to the Beltrami equation}

Let $D$ be a domain in the complex plane $\mathbb{C}$, i.e., a connected and open subset of $\mathbb{C}$, and let $\mu: D \rightarrow \mathbb{C}$ be a measurable function with $|\mu(z)|<1$ a.e. in $D$. The Beltrami equation looks like this:

$$
f_{\bar{z}}=\mu(z) f_{z},
$$

where $f_{\bar{z}}=\bar{\partial} f=\left(f_{x}+i f_{y}\right) / 2, f_{z}=\partial f=\left(f_{x}-i f_{y}\right) / 2, z=x+i y, f_{x}$ and $f_{y}$ are the partial derivatives of $f$ with respect to $x$ and $y$. The function $\mu$ is called the complex coefficient, and

$$
K_{\mu}(z)=\frac{1+|\mu(z)|}{1-|\mu(z)|}
$$

is the dilation ratio of equation (114). A Beltrami equation (114) is said to be degenerate if $K_{\mu}(z)$ is essentially unbounded, i.e., $K_{\mu}(z) \notin L^{\infty}(D)$.

Below we present a theorem on the relationship between the homeomorphic $W_{\text {loc }}^{1,1}$-solutions of the Beltrami equation and the lower $Q$-homeomorphisms (see, e.g., [20, Theorem 1]).

Theorem 12. Let $f$ be a homeomorphic solution of class $W_{\mathrm{loc}}^{1,1}$ for the Beltrami equation (114). Then $f$ is a lower $Q$-homeomorphism at each point $z_{0} \in D$ with $Q(z)=K_{\mu}(z)$.

Combining Theorem 12 with Theorem 8 for $p=2$, we arrive at the following result.

Theorem 13. Let $f: \mathbb{B} \rightarrow \mathbb{B}$ be a homeomorphic $W_{\text {loc }}^{1,1}$-solution of the Beltrami equation (114), and let $f(0)=0$. Then

$$
\liminf _{z \rightarrow 0}|f(z)| \cdot \exp \left\{2 \pi \int_{|z|}^{1} \frac{d t}{\left\|K_{\mu}(z)\right\|_{1}(t)}\right\} \leq 1,
$$

where $\left\|K_{\mu}(z)\right\|_{1}(r)=\int_{C(0, r)} K_{\mu}(z)|d z|$ and $C(0, r)=\{z \in \mathbb{C}:|z|=r\}$.

Corollary 4. In particular, if $K_{\mu}(z) \leq K$ for a.e. $z \in \mathbb{B}$, then

$$
\liminf _{z \rightarrow 0} \frac{|f(z)|}{|z|^{1 / K}} \leq 1 \text {. }
$$




\section{REFERENCES}

[1] M. A. Lavrent'ev, Variational methods for boundary value problems for systems of elliptic equations, Izdat. Akad. Nauk SSSR, Moscow, 1962; English transl., Noordhoff, Ltd., Groningen, 1963. MR0146377 (26:3899)

[2] O. Martio, V. Ryazanov, U. Srebro, and E. Yakubov, Moduli in modern mapping theory, Springer Monogr. Math., Springer, New York etc., 2009. MR2466579 (2012g:30004)

[3] B. Fuglede, Extremal length and functional completion, Acta Math. 98 (1957), 171-219. MR.0097720 $(20: 4187)$

[4] F. W. Gehring, Rings and quasiconformal mappings in space, Trans. Amer. Math. Soc. 103 (1962), 353-393. MR0139735 (25:3166)

[5] V. I. Ryazanov and E. A. Sevost'yanov, Equicontinuous classes of ring Q-homeomorphisms, Sibirsk. Mat. Zh. 48 (2007), no. 6, 1361-1376; English transl., Sib. Mat. J. 48 (2007), no. 6, 1093-1105. MR2397516(2009k:30030)

[6] R. R. Salimov, Absolute continuity on lines and the differentiability of a generalization of quasiconformal mappings, Izv. Ross. Akad. Nauk Sci. Mat. 72 (2008), no. 5, 141-148; English transl., Izv. Math. 72 (2008), no. 5, 977-984. MR2473774 (2010a:30038)

[7] Estimation of the measure of the image of the ball, Sibirsk. Mat. Zh. 53 (2012), no. 4, 920-930; English transl., Sib. Math. J. 53 (2012), no. 4, 739-747. MR3013536

[8] _ , On finitely Lipschitz space mappings, Sib. Elektron. Mat. Izv. 8 (2011), $284-295$. $\operatorname{MR} 2876547(2012 \mathrm{~m}: 30036)$

[9] - On the Lipschitz property of a class of mappings, Mat. Zametki 94 (2013), no. 4, 591-599; English transl., Math. Notes 94 (2013), no. 4, 559-566.

[10] R. R. Salimov and E. A. Sevost'yanov, Theory of ring Q-mappings and geometric function theory, Mat. Sb. 201 (2010), no. 6, 131-158; English transl., Sb. Math. 201 (2010), no. 5-6, 909-934. MR.2682368(2011i:30026)

[11] E. A. Sevost'yanov, On the theory of the removal of singularities for mappings with an unbounded characteristic of quasiconformality, Izv. Ross. Akad. Nauk Ser. Mat. 74 (2010), no. 1, 159-174; English transl., Izv. Math. 74 (2010), no. 1, 151-165. MR2655240 (2011c:30066)

[12] _ On space mappings with integral restrictions on the characteristic, Algebra i Analiz 24 (2012), no. 1, 131-156; English transl., St. Petersburg Math. J. 24 (2013), no. 1, 99-115. MR3013296

[13] - On the branch points of mappings with an unbounded characteristic of quasiconformality, Sibirsk. Mat. Zh. 51 (2010), no. 5, 1129-1146; English transl., Sib. Math. J. 51 (2010), no. 5, 899-912. MR2768509 (2011k:30027)

[14] A. Golberg, Homeomorphisms with integrally restricted moduli, Complex Analysis and Dynamical Systems. IV, Pt. 1, Contemp. Math., vol. 553, Amer. Math. Soc., Providence, RI, 2011, pp. 83-98. MR.2868590

[15] V. I. Kruglikov, Capacities of condensors and quasiconformal in the mean mappings in space, Mat. Sb. 130 (1986), no. 2, 185-206; English transl., Sb. Math. 58 (1987), no. 1, 185-205. MR854971 (88d:30028)

[16] S. K. Vodop'yanov and A. D. Ukhlov, Superposition operators in Sobolev spaces, Izv. Vyssh. Uchebn. Zaved. Mat. 2002, no. 10, 11-33; English transl., Russian Math. (Iz. VUZ) 46 (2002), no. 10, 9-31. MR 1956487 (2003m:47044)

[17] Superposition operators in Lebesgue spaces and the differentiability quasi-additive set functions, Vladikavkaz. Mat. Zh. 4 (2002), no. 1, 11-33. (Russian) MR2065083 (2005b:28006)

[18] S. K. Vodop'yanov, Description of substitution operators of Sobolev spaces, Dokl. Akad. Nauk 400 (2005), no. 1, 11-15; English transl., Dokl. Math. 71 (2005), no. 1, 5-9. MR2159591(2008c:47047)

[19] Composition operators on Sobolev spaces, Complex Analysis and Dynamical Systems. II, Contemp. Math., vol. 382, Amer. Math. Soc., Providence, RI, 2005, pp. 401-415. MR2175907 (2006g:46058)

[20] D. A. Kovtonyuk, I. V. Petkov, V. I. Ryazanov, and R. R. Salimov, Boundary behavior and the Dirichlet problem for the Beltrami equations, Algebra i Analiz 25 (2013), no. 4, 101-124; English transl., St. Petersburg Math. J. 25 (2014), no. 4, 587-603. MR3184618

[21] T. Lomako, R. Salimov, and E. A. Sevost'yanov, On equicontinuity of solutions to the Beltrami equations, Ann. Univ. Buchar. Math. Ser. 1 (LIX) (2010), no. 2, 263-274. MR2920236

[22] V. I. Ryazanov, R. R. Salimov, U. Srebro, and E. Yakubov, On boundary value problems for the Beltrami equations, Complex Analysis and Dynamical Systems. IV, Contemp. Math., vol. 591, Amer. Math. Soc., Providence, RI, pp. 211-242. MR3155690

[23] E. S. Afanas'eva, V. I. Ryazanov, and R. R. Salimov, On mappings in Orlicz-Sobolev classes on Riemannian manifolds, Ukr. Mat. Visn. 8 (2011), no. 3, 319-342; English transl., J. Math. Sci. (N.Y.) 181 (2012), no. 1, 1-17. MR2906695 
[24] D. A. Kovtonyuk, V. I. Ryazanov, R. R. Salimov, and E. A. Sevost'yanov, On the theory of OrliczSobolev classes, Algebra i Analiz 25 (2013), no. 6, 50-102; English transl., St. Petersburg Math. J. 25 (2014), no. 6, 929-963. MR3234840

[25] D. A. Kovtonyuk, V. I. Ryazanov, R. R. Salimov, and E. A. Sevost'yanov, On mappings in the Orlicz-Sobolev classes, Ann. Univ. Buchar. Math. Ser. 3(LXI) (2012), no. 1, 67-78. MR3034963

[26] O. Martio, S. Rickman, and J. Väisälä, Definitions for quasiregular mappings, Ann. Acad. Sci. Fenn. Ser. A1. Math. 448 (1969), 1-40. MR0259114(41:3756)

[27] J. Hesse, A p-extremal length and p-capacity equality, Ark. Mat. 13 (1975), 131-144. MR0379871 $(52: 776)$

[28] V. A. Shlyk, On the equality between p-capacity and p-modulus, Sibirsk. Mat. Zh. 34 (1993), no. 6, 216-221; English transl., Sib. Mat. J. 34 (1993), no. 6, 1196-1200. MR1268174 (95d:31003)

[29] F. W. Gehring, Quasiconformal mappings, Complex Analysis and its Applications. Vol. II, Internat. Atomic Energy Agency, Vienna, 1976, pp. 213-268. MR0480997 (58:1144)

[30] V. M. Gol'dshtein and Yu. G. Reshetnyak, Introduction to the theory of functions with generalized derivatives and quasiconformal mappings, Nauka, Moscow, 1983. (Russian) MR738784 $(85 \mathrm{~m}: 46031 \mathrm{a})$

[31] V. G. Maz'ya, Classes of domains measures and capacities in the theory of spaces of differentiable functions, Current problems in mathematics. Fundamental directions, vol. 26, Itogi Nauki i Tekhniki, Akad. Nauk SSSR, VINITI, Moscow, 1988, pp.159-228. (Russian) MR,1178112

[32] S. Saks, Theory of the integral, 2nd ed., Dover Publ., New York, 1964. MR0167578(29:4850)

[33] D. A. Kovtonyuk and V. I. Ryazanov, On the theory lower Q-homeomorphisms, Ukr. Mat. Visn. 5 (2008), no. 2, 159-184; English transl., Urk. Math. Bull. 5 (2008), no. 2, 157-181. MR2559832 (2010k:30022)

[34] W. P. Ziemer, Extremal length and p-capacity, Michigan Math. J. 16 (1969), 43-51. MR0247077 (40:346)

[35] T. Ransford, Potential theory in the complex plane, London Math. Soc. Student Text, vol. 28, Cambridge Univ. Press, Cambridge, 1995. MR.1334766 (96e:31001)

[36] T. Rado and P. V. Reichelderfer, Continuous transformations in analysis, Grundlehren. Math. Wiss., Bd. 75, Springer-Verlag, Berlin, 1955. MR0079620 (18:115c)

[37] H. Federer, Geometric measure theory, Grundlehren. Math. Wiss., Bd. 153, Springer, Berlin etc., 1969. MR0257325 (41:1976)

[38] F. W. Gehring and O. Lehto, On the total differentiability of functions of a complex variable, Ann. Acad. Sci. Fenn. Ser. A1. Math. 272 (1959), 3-8. MR0124487(23:A1799)

[39] F. W. Gehring, Lipschitz mappings and the p-capacity of ring in n-space, Advances Theory Riemann Surfaces (Proc. Conf. Stonybrook, N.Y., 1969), Ann. of Math. Stud., vol. 66, Princeton Univ. Press, Princeton, NJ, 1971, pp. 175-193. MR0310238(46:9340)

[40] T. Iwaniec and G. Martin, Geometric function theory and non-linear analysis, Oxford Math. Monogr., Clarendon Press, Oxford, 2001. MR1859913(2003c:30001)

[41] T. Iwaniec and V. Sverák, On mappings with integrable dilatation, Proc. Amer. Math. Soc. 118 (1993), no. 1, 181-188. MR.1160301 (93k:30023)

[42] D. A. Kovtonyuk and V. I. Ryazanov, On the theory of mappings with finite area distortion, J. Anal. Math. 104 (2008), 291-306. MR2403438 (2009e:30042)

[43] V. G. Maz'ya, Sobolev spaces, Leningrad. Univ., Leningrad, 1985; English transl., Springer Ser. Soviet Math., Springer, Berlin, 1985. MR0807364 (87g:46055) MR0817985 (87g:16056)

[44] D. Menchoff, Sur les differencelles totales des fonctions univalentes, Math. Ann. 105 (1931), no. 1, 75-85. MR1512705

Institute of Applied Mathematics and Mechanics, National Academy of Science, 74 Rosa Luksemburg Str., 83114 Donetzk, Ukraine

E-mail address: ruslan623@yandex.ru

Received 23/JAN/2014

Translated by A. PLOTKIN 